\title{
The Association Between Body Composition and Serum Glucose, Lipids, Leptin, and Insulin in a Health-Check Population
}

\author{
Mingkun Zhang ${ }^{1}$, Lin Sun ${ }^{2}$, Weixiang Wang ${ }^{2}$, Nana $\mathrm{Li}^{1}$, Qianwen Li ${ }^{1}$, Ling Wang ${ }^{1}$, * \\ ${ }^{1}$ College of Public Health, Zhengzhou University, Zhengzhou, Henan, China \\ ${ }^{2}$ Affiliated Renmin Hospital, Zhengzhou University, Zhengzhou, Henan, China
}

\section{Email address:}

1298842984@qq.com (Mingkun Zhang), zsunlin@sina.com (Lin Sun), lisalingwang@zzu.edu.cn (Ling Wang)

\section{To cite this article:}

Mingkun Zhang, Lin Sun, Weixiang Wang, Nana Li, Qianwen Li, Ling Wang. The Association Between Body Composition and Serum Glucose, Lipids, Leptin, and Insulin in a Health-Check Population. American Journal of Clinical and Experimental Medicine.

Vol. 3, No. 5, 2015, pp. 293-299. doi: 10.11648/j.ajcem.20150305.27

\begin{abstract}
To study the association between body composition and serum glucose, lipids, leptin, and insulin in a group of health-check population. Total of 205 subjects under routine health check were investigated. After overnight fasting, they were checked for body composition, serum glucose, lipids (triglyceride, total cholesterol, high-density lipoprotein cholesterol, low-density lipoprotein cholesterol (LDL-C)), leptin, and insulin. Because of the seriously skewed distribution of leptin and insulin data, their natural logarithm, named as Lnlep and Lnins were chosen in data analysis. result: Male subjects at all age groups showed higher body weight, height, waist circumstance, BMI, percentages of muscle mass, water and mineral components, LDL-C, and overweight rate then that in female (all $\mathrm{P}<0.05$ ); With the increase of age, both genders showed decreased height, percentages of muscle mass, water and protein components $(\mathrm{P}<0.05$ for both), but increased fasting blood glucose (FBG) and overweight rate $(\mathrm{P}<0.05$ for both). As for females, their mean weight, total fat mass, and percentage of fat mass were increased along age increase (all $\mathrm{P}<0.05)$; The impact factors of BMI in these population were waist circumstance, percentage of fat mass, Lnins, and gender. Their standardized partial regression coefficients were $0.650,0.358,0.082$, and 0.103 respectively (all $\mathrm{P}<0.05$ ). Conclusion: ody weight, waist circumstance, BMI, serum LDL-C, and are positively associated with age; Waist circumstance, percentage of body fat, and serum insulin were positively associated with BMI.
\end{abstract}

Keywords: Health-Check, Body Composition, Leptin, Insulin

\section{人体成分与血液生化指标及部分激素水平的关系}

\author{
张明坤 ${ }^{1}$, 孙琳 ${ }^{2}$, 王卫香 ${ }^{2}$, 李娜娜 ${ }^{1}$, 李倩文 ${ }^{1}$, 王玲 ${ }^{1, *}$ \\ 郑州大学公共卫生学院, 郑州, 河南, 中国 \\ 郑州大学附属人民医院, 郑州, 河南, 中国
}

\section{邮箱}

1298842984@qq.com（张明坤）, zsunlin@sina.com（孙琳）, lisalingwang@zzu.edu.cn（王玲）

摘要: 分析体成分与血生化指标及部分激素水平的关系。选取某体检人群 205 人, 测体成分、空腹血糖、血脂、瘦素及胰岛 素水平。取瘦素及胰岛素结果的自然对数进行分析，分析不同年龄、性别之间BMI与体成分、血糖血脂以及部分激素水平的 关系。结果: 男性各年龄组身高、体重、腰围、BMI, 水分量、肌肉量和矿物质百分比, 血LDL-C及超重率均高于女性 $(P<0.05)$; 随着年龄增加, 该人群身高、水分量、肌肉量和身体蛋白质百分比降低, 空腹血糖以及超重率逐渐升高（男女P均 $<0.05 ）$; 女性体重、腰围、体脂肪率、体脂肪量随年龄而增加（P均 $<0.05 ）$; 人群BMI的主要影响因素为腰围、体脂肪率、胰岛素的 自然对数及性别, 其标准化偏回归系数分别为 $0.650 、 0.358 、 0.082 、 0.103$ （P均 $<0.05 ） 。$ 结论：体重、腰围、BMI、血清 LDL-C、空腹血糖与年龄呈正相关; 腰围、体脂肪率以及血胰岛素与BMI呈正相关。

关键词: 体检人群, 体成分, 瘦素, 胰岛素 


\section{1. 引言}

近年来, 人们的生活水平逐渐提高, 饮食习惯和劳 作方式也以省力便捷为主, 这一生活方式使得体力活动 减少, 从而脂肪易堆积等问题也越来越突出, 肥胖及其 相关并发症已成为全球医疗卫生领域关注的热点问题, 目前, 有关人体体成分、代谢相关激素水平及血液生化 指标与肥胖关系的研究较多, 但大部分集中在某一年龄 段或者某一性别的研究, 很少将年龄、性别等因素分类 进行探讨, 本研究以此为出发点, 研究不同年龄、不同 性别人群的体成分、血液生化及激素等指标的差异性, 探究发生肥胖的相关因素。

\section{2. 研究设计}

\section{1. 研究对象}

利用简单随机方法选取 2013 年 10 月在河南省人民 医院进行体检的某体检人群 205 例为研究对象。纳入标 准：1）有代谢综合征组分, 满足中心性肥胖、糖耐量 异常、甘油三酯升高、高密度脂蛋白胆固醇降低以及高 血压中其中一项或两项者；2）无其他并发症，包括心 血管疾病以及二型糖尿病等； 3 ）自愿参加本研究并签 署知情同意书者。排除标准：1）排除身体有重大疾病, 传染病, 原发性疾病者；2）排除机体处于特殊状态, 如女性处于妊娠期以及各种原因造成水肿等不适宜进 行体成分检查者。经郑州大学生命科学伦理审查委员会 审查, 该项目研究内容和过程遵循国际及国家颁布的有 关生物医学研究的伦理要求。

\section{2. 研究方法}

\subsection{1. 体检人群体成分的测量}

使用韩国杰文DX-200人体成分分析仪测量研究对象 的体重、身高、身体质量指数（Body mass index，BMI）、 体脂肪量、肌肉量、水分量、蛋白质、无机盐含量等体成 分指标。要求受试者空腹、脱鞋袜、着单衣。为了排除体 重对于各体成分的影响, 使体成分指标直接可比, 比较时 使用水分量百分比, 肌肉量百分比, 蛋白质百分比以及矿 物质百分比。

水分量百分比 $=\frac{\text { 水分量 }(\mathrm{Kg})}{\text { 体重 }(\mathrm{Kg})} \times 100 \%$, 即水分量占体重 的百分比。同理, 求出其他成分的百分比, 进行各指标间 比较。

\subsection{2. 体检人群超重者的判断}

根据前卫生部《中国成人超重与肥胖症预防控制指南》 ${ }^{[1]}$ 推荐的标准。BMI $<18.5 \mathrm{Kg} / \mathrm{m}^{2}$ 者属于体重偏低, BMI18. $5^{\sim} 23.9$ 为正常, BMI24. 0 27.9 为超重, BMI $\geqslant 28.0$ 者为肥胖。按此标准, 本次研究以 $B M I=24.0 \mathrm{Kg} / \mathrm{m}^{2}$ 为截断 值, 体检人群中BMI $\geqslant 24.0$ 者为超重者, 其余为非超重者。

\subsection{3. 体检人群中心性肥胖的判断}

《中国成人血脂异常防治指南》 ${ }^{[2]}$ 制订联合委员会对 代谢综合征制订了组分量化标准。其中关于中心性肥胖做 出如下规定: 腰围 $\geqslant 90 \mathrm{~cm}$ (男性) 或 $\geqslant 80 \mathrm{~cm}$ (女性)。

按照上述标准, 本次研究以男性腰围 $90 \mathrm{~cm}$, 女性腰围 $80 \mathrm{~cm}$ 为截断值, 将男性腰围 $\geqslant 90 \mathrm{~cm}$ 者、女性腰围 $\geqslant 80 \mathrm{~cm}$ 者定义为中心性肥胖。

\subsection{4. 体检人群血糖血脂及激素指标的检测}

于清晨空腹状态采集上述体检人群静脉血 $5 \mathrm{~m} 1$, $3000 \mathrm{rpm}, 4^{\circ} \mathrm{C}$ 离心 $10 \mathrm{~min}$ 分离血清。利用人瘦素酶联免疫 试剂盒 (上海抚生实业有限公司), 通过酶联免疫双抗体 夹心法检测血清瘦素水平; 利用胰岛素测定试剂盒 (Beckman Coutler, Inc.) , 通过化学发光法检测血清 胰岛素水平, 以上指标的测定均按照试剂盒说明书严格进 行操作。体检人群的空腹血糖、 TG、TC、HDL-C、 LDL-C 等检测结果由河南省人民医院检验科提供。

\section{3. 数据分析}

所有数据均用SPSS17.0统计学软件包进行分析, 计 量资料采用平均值士标准差 $(\bar{x} \pm \mathrm{s})$ 表示, 计数资料采 用个数 (百分率\%) 表示。对于计量资料, 进行不同性别 比较时, 采取独立样本 $t$ 检验; 进行年龄组的比较时, 采 取单因素方差分析, 组间两两比较使用LSD法。对于血清 瘦素与胰岛素, 由于资料严重偏态, 故在分析前先进行 对数化处理, 对瘦素及胰岛素取自然对数 (分别计为 Lnlep和Lnins) 后进行分析, 检验水准 $P<0.05$ 为差异显 著, $P<0.01$ 为差异极显著。对于计数资料进行不同性别 以及年龄组率比较时, 使用 $x^{2}$ 检验, 检验水准同上; 不 同年龄组中率进行两两比较时, 采用Bonferroni法, 校 正检验水准 $a^{\prime}=a / m$, 其中 $m$ 为两两比较次数。进行回 归分析时, 采用多元线性回归, 以BMI为因变量, 自变量 篮选采用逐步法（stepwise）。

\section{3. 结果}

\section{1. 体检人群的一般情况}

本次研究对象共计 205 人, 其中男性 78 人, 年龄 $(35.5 \pm 10.2)$ 岁, 女性 127 人, 年龄 $(35.5 \pm 10.5)$ 岁 $(\mathrm{P}=0.965)$ 。

各年龄组研究对象的性别分布如表1所示,

表1 体检人群的性别年龄分布 $[\mathrm{n}(\%)]$ 。

\begin{tabular}{lll}
\hline 年龄组 & 男性 & 女性 \\
\hline $20^{\sim}$ & $31(40.8 \%)$ & $45(59.2 \%)$ \\
$30^{\sim}$ & $19(29.4 \%)$ & $48(71.6 \%)$ \\
$40^{\sim}$ & $28(45.2 \%)$ & $34(54.8 \%)$ \\
\hline
\end{tabular}

$x^{2}=4.242, \quad P=0.120$ 


\section{2. 不同性别年龄体检人群体成分比较}

\subsection{1. 不同性别体检人群体成分比较}

1) 20 岁组体检人群男女体成分比较

20 岁年龄组男性身高、体重、腰围、体脂肪量、BMI、 水分量百分比、肌肉量百分比以及蛋白质百分比等指标显 著高于女性 $(P$ 均 $<0.01)$; 体脂肪量和矿物质百分比也显
著高于女性（ $P$ 均 $<0.05 ）$; 女性体脂肪率虽高于男性, 但 差异无统计学意义 $(P>0.05)$ 。

2) $30^{\sim}$ 岁组体检人群男女体成分比较

30 岁年龄组男性身高、体重、腰围、BMI均显著高于 女性 $(\mathrm{P}$ 均 $<0.01)$; 体脂肪量、水分量百分比、肌肉量百 分比、矿物质百分比也显著高于女性（均 $\mathrm{P}<0.05 ）$; 女性 体脂肪率与蛋白质百分比高于男性, 但差异无统计学意义, 与蛋白质百分比高于男性, 但差异无统计学意义 $(P>0.05)$ 。

表2 不同性别与年龄组体成分状况 $(\bar{x} \pm s)$ 。

\begin{tabular}{|c|c|c|c|c|c|c|c|c|}
\hline 年龄组 & 性别 & $n$ & 身高 $(\mathrm{cm})$ & 体重（Kg） & 腰围 $(\mathrm{cm})$ & 体脂肪率（\%) & 体脂肪量 $(\mathrm{Kg})$ & BMI $\left(\mathrm{Kg} / \mathrm{m}^{2}\right)$ \\
\hline \multirow{2}{*}{$20^{2}$} & 男 & 31 & $174.8 \pm 4.9^{a a}$ & $73.9 \pm 9.5^{\text {aа }}$ & $85.1 \pm 7.0^{\text {aa }}$ & $24.0 \pm 5.3$ & $17.4 \pm 5.0^{\mathrm{a}}$ & $24.1 \pm 2.5^{\text {aa }}$ \\
\hline & 女 & 45 & $162.4 \pm 3.9$ & $55.1 \pm 7.5$ & $70.4 \pm 6.9$ & $26.2 \pm 5.0$ & $14.6 \pm 4.4$ & $20.9 \pm 2.7$ \\
\hline \multirow{2}{*}{$30^{\sim}$} & 男 & 19 & $172.5 \pm 5.3^{\text {aa }}$ & $75.4 \pm 12.3^{\text {aа }}$ & $87.8 \pm 10.7^{\text {aa }}$ & $25.3 \pm 5.5$ & $19.6 \pm 7.0^{\mathrm{a}}$ & $25.3 \pm 3.5^{\text {aa }}$ \\
\hline & 女 & 48 & $162.4 \pm 4.4$ & $57.7 \pm 7.9$ & $73.6 \pm 7.4$ & $27.8 \pm 4.2$ & $16.5 \pm 4.4$ & $21.8 \pm 2.5$ \\
\hline \multirow{2}{*}{$40^{\sim}$} & 男 & 28 & $171.6 \pm 3.5^{* a \mathrm{a}}$ & $76.4 \pm 9.6^{\text {aa }}$ & $89.5 \pm 9.8^{\text {aа }}$ & $26.7 \pm 3.9^{\text {aа }}$ & $20.3 \pm 5.7$ & $26.0 \pm 3.4^{*_{a}}$ \\
\hline & 女 & 34 & $160.3 \pm 3.8^{* *}$ & $60.7 \pm 9.4^{* * *}$ & $78.7 \pm 8.3^{* * * \# \#}$ & $30.2 \pm 5.1^{\text {w*\# }}$ & 19. $2 \pm 5.7^{\text {w*\# }}$ & $23.6 \pm 3.7^{\text {wexth }}$ \\
\hline
\end{tabular}

表3 不同性别与年龄组血液激素指标状况 $(\bar{x} \pm s)$ 。

\begin{tabular}{lllllllll}
\hline 年龄组 & 性别 & HDL (mmol/L) & LDL (mmol/L) & TG (mmol/L) & TC (mmol/L) & FBG (mmol/L) & Lnlep & Lnins \\
\hline $20^{\sim}$ & 男 & $1.23 \pm 0.21^{\text {aa }}$ & $2.93 \pm 0.63^{\text {aa }}$ & $1.43 \pm 0.85^{\text {aa }}$ & $4.64 \pm 0.76$ & $5.12 \pm 0.36$ & $2.60 \pm 0.82$ & $1.79 \pm 0.50$ \\
& 女 & $1.54 \pm 0.31$ & $2.45 \pm 0.53$ & $0.85 \pm 0.40$ & $4.44 \pm 0.64$ & $4.97 \pm 0.58$ & $2.76 \pm 0.76$ & $1.70 \pm 0.55$ \\
$30^{\sim}$ & 男 & $1.30 \pm 0.40$ & $2.89 \pm 0.85^{\mathrm{a}}$ & $1.34 \pm 0.62$ & $4.76 \pm 1.16$ & $5.21 \pm 0.43$ & $2.72 \pm 0.68$ & $2.06 \pm 0.59^{\mathrm{a}}$ \\
& 女 & $1.46 \pm 0.21$ & $2.60 \pm 0.64$ & $1.05 \pm 0.60$ & $4.52 \pm 0.77$ & $4.99 \pm 0.40$ & $2.61 \pm 0.90$ & $1.61 \pm 0.90$ \\
$40^{\sim}$ & 男 & $1.24 \pm 0.20^{\text {aa }}$ & $3.19 \pm 0.69^{\text {aa }}$ & $1.64 \pm 0.66^{\text {aa }}$ & $5.02 \pm 0.84$ & $5.98 \pm 2.27^{\text {a* }}$ & $2.30 \pm 0.42$ & $1.86 \pm 0.57$ \\
& 女 & $1.51 \pm 0.30$ & $2.79 \pm 0.73$ & $1.07 \pm 0.52$ & $4.83 \pm 1.08$ & $5.27 \pm 0.56^{\text {** }}$ & $2.49 \pm 0.80$ & $1.58 \pm 0.55$ \\
\hline
\end{tabular}

注: 与 $20^{\sim}$ 岁年龄组相比, ${ }^{*} P<0.05,{ }^{* *} P<0.01$ 。与 $30^{\sim}$ 岁年龄组相比, ${ }^{*} P<0.05,{ }^{* \prime} P<0.01$ 。与女性相比 $P<0.05,{ }^{a a} P<0.01$ 。

3) $40^{\sim}$ 岁组体检人群男女体成分比较

40 岁以上年龄组男性身高、体重、腰围、体脂肪率、 水分量百分比、肌肉量百分比、蛋白质百分比、矿物质百 分比均显著高于女性（P均 $<0.01 ） ; B M I 也$ 显著高于女性 $(P<0.05)$; 体脂肪量无性别差异 $(P>0.05)$ 。结果详见 表2及图1。

\subsection{2. 不同年龄体检人群体成分比较}

1) 男性体检人群体成分随年龄变化的趋势

随着年龄的增大, 男性平均身高、水分量百分比、肌 肉量百分比、以及蛋白质百分比逐渐降低（P均<0.05）; 其平均体重、腰围、体脂肪率、体脂肪量、BMI等指标呈 逐渐上升趋势, 而矿物质百分比有降低趋势, 但均无统计 学意义 $(P>0.05)$ 。

2) 女性体检人群体成分随年龄变化的趋势

随着年龄组的增加, 女性平均身高, 水分量百分比、 蛋白质百分比、肌肉量百分比逐渐降低 $(\mathrm{P}$ 均 $<0.01)$; 平 均体重、腰围、体脂肪率、体脂肪量、BMI逐渐升高 (
均 $<0.05)$ : 矿物质百分比有降低趋势, 但无统计学意义 (P>0.05)。结果详见图1、表2。

\section{3. 不同性别年龄体检人群血生化与激素指标比较}

\section{3.1. 不同性别体检人群血生化与激素指标的比较}

1) $20^{\sim}$ 岁组体检人群男女血液检测指标比较

在 $20^{\sim}$ 岁年龄组, 男性血清 HDL-C水平显著低于女性 $(\mathrm{P}<0.01)$, 血清 $\mathrm{LDL}-\mathrm{C}$ 以及 $\mathrm{TG}$ 水平显著高于女性（P均 $<0.01 ） ;$ TC、FBG、Lnins高于女性, 而Lnlep低于女性, 但差异无统计学意义（P均>0.05）。

2) 30 岁组体检人群男女血液检测指标比较

30 岁年龄组男性血清LDL-C和Lnins显著高于女性 (P 均 $<0.05 ）, T G 、 T C 、 F B G 、 L n 1 e p$ 高于女性, 而HDL-C低于 女性, 但差异无统计学意义（P均>0.05）。

3) 40 岁组体检人群男女血液检测指标比较

40 岁年龄组男性血清HDL-C显著低于女性 $(\mathrm{P}<0.01)$, TG、LDL、FBG显著高于女性（P均 $<0.05 ）$ ，血清TC和Lnins 
高于而Lnlep低于女性, 但差异无统计学意义 ( $P>0.05) 。$
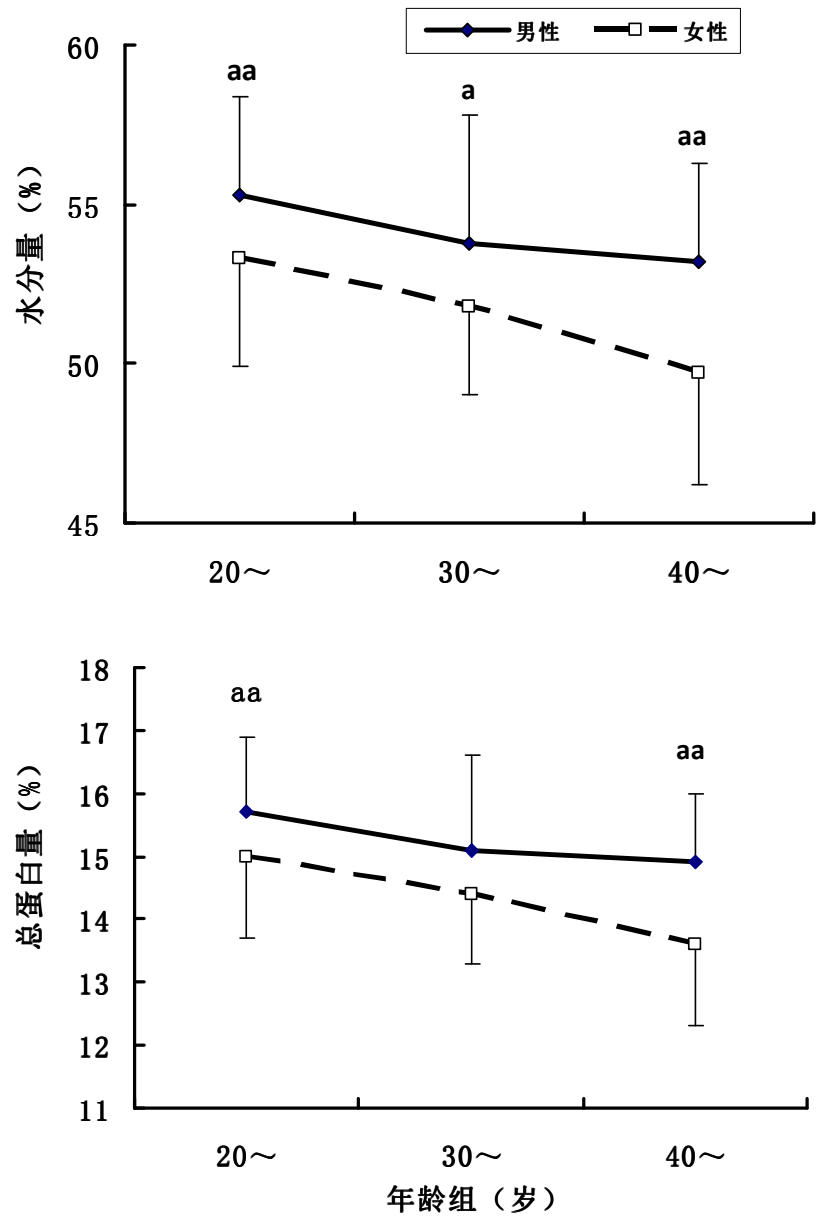

结果详见表3。
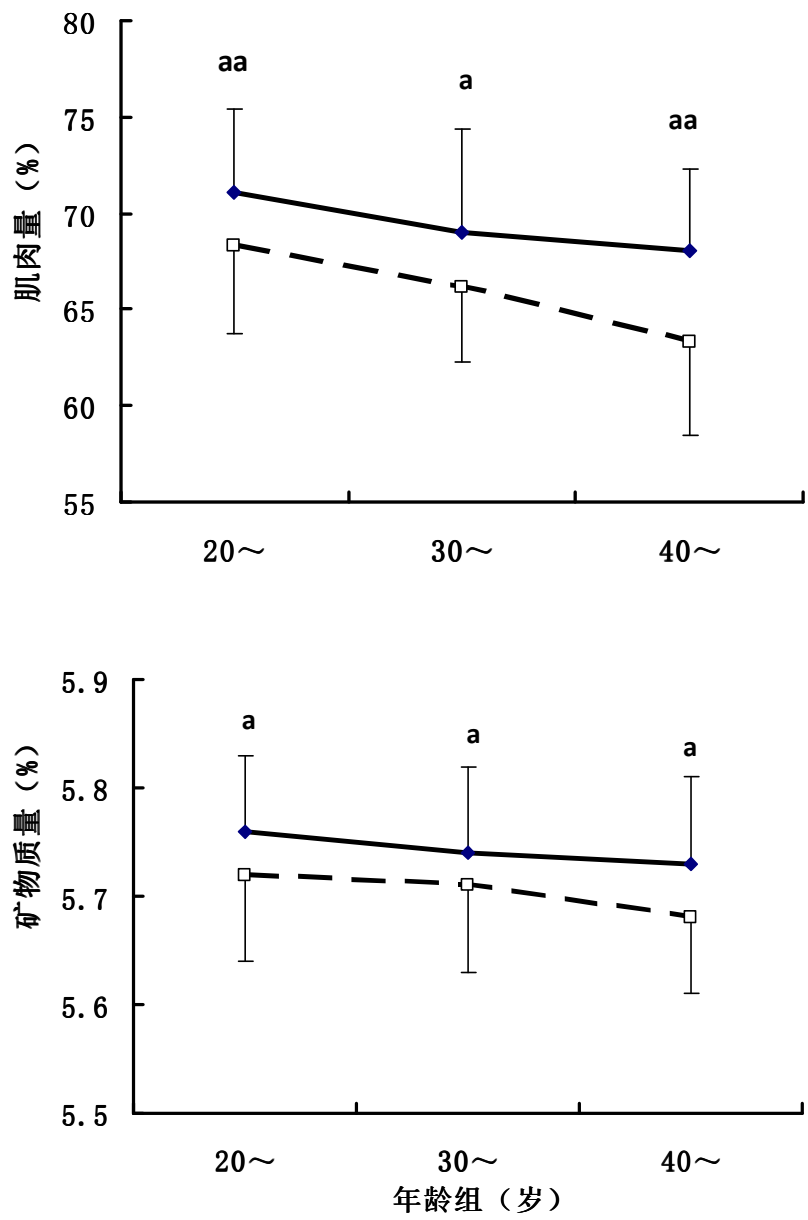

注: 与女性相比, ${ }^{\mathrm{a}} P<0.05,{ }^{\mathrm{aa}} P<0.01$ 。

图1 不同性别年龄体检人群体成分比较。

\subsection{2. 不同年龄体检人群血生化与激素指标的比较}

1) 男性体检人群血液生化以及激素指标随年龄变化 趋势

男性体检人群，随着年龄的增加，其平均FBG逐渐升 高 $(P<0.05) ， T C ， L D L$ 有上升趋势，但差异无显著性 (P>0.05) ; 其平均 TG、HDL-C、Lnlep、Lnins先下降后 上升，但差异无显著性（P>0.05）。 趋势

2) 女性体检人群血液生化以及激素指标随年龄变化

女性随着年龄的增加, 其平均FBG逐渐升高 $(\mathrm{P}<0.05)$ 、 TG、TC、LDL有上升趋势但差异无显著性 $(P>0.05)$; 平 均Lnlep和Lnins 有下降趋势, 但差异无显著性 $(P>0.05)$ 。 详见表4。

\section{4. 体检人群超重以及腰围超标状况}

\section{4. 1. 体检人群超重情况}

1)不同性别体检人群超重情况

男性各个年龄组超重率都显著高于女性, 分别为: $20^{\sim}$ 岁组, 男性 $38.1 \%$ vs. 女性 $13.3 \%$; $30^{\sim}$ 岁组, 男性 $68.4 \%$ vs.
女性 $16.7 \%$; $40^{\sim}$ 岁组, 男性 $78.6 \%$ vs. 女性 $41.2 \%$ （P均 <0.01）。详见表4。

表4 各年龄组不同性别超重情况 [n（\%）]。

\begin{tabular}{ll|llll}
\hline 年龄组 & 性别 & 未超重者 & 超重者 & $x^{2}$ & $P$ \\
\hline $20^{\sim}$ & 男 & $13(61.9 \%)$ & $8(38.1 \%)$ & & \\
& 女 & $39(86.7 \%)$ & $6(13.3 \%)$ & & 0.000 \\
$30^{\sim}$ & 男 & $6(31.6 \%)$ & $13(68.4 \%)$ & & \\
& 女 & $40(83.3 \%)$ & $8(16.7 \%)$ & & 0.000 \\
$40^{\sim}$ & 男 & $6(21.4 \%)$ & $22(78.6 \%)$ & & \\
& 女 & $20(58.8 \%)$ & $14(41.2 \%)$ & & 0.003 \\
\hline
\end{tabular}

2)不同年龄组体检人群超重情况

男性随着年龄增加, 其超重率逐渐上升, 分别为: $20^{\sim}$ 岁组 $38.1 \%, 30^{\sim}$ 岁组 $68.4 \%, 40^{\sim}$ 岁组 $78.6 \%\left(x^{2}=8.761\right.$, $\mathrm{P}=0.013$ ）。女性随着年龄增加, 其超重率也逐渐上升, 分别为: $20^{\sim}$ 岁组 $13.3 \%, 30^{\sim}$ 岁组 $16.7 \%, 40^{\sim}$ 岁组 $41.2 \%$ $\left(x^{2}=10.036, P=0.007\right)$ 。具体情况如图2所示。 


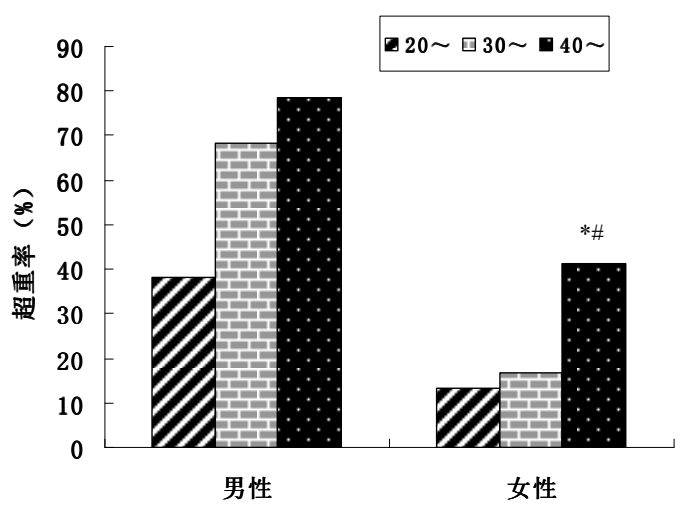

注: 与 $20^{\sim}$ 岁组比, $* \mathrm{P}=0.0167$; 与 $30^{\sim}$ 岁组比, $\# \mathrm{P}=0.0167$ 。

图2 男女体检人群不同年龄组超重情况。

\section{4. 2. 体检人群中心性肥胖发生情况}

1) 不同性别体检人群中心性肥胖发生情况

在 $20^{\sim}$ 岁和 $30^{\sim}$ 岁年龄组, 男性中心性肥胖发生率显著 高于女性, 分别为: $20^{\sim}$ 岁组, 男性 $32.3 \%$ vs. 女性 $8.9 \%$; 30 岁组 男性 $47.4 \%$ vs. 女性 $22.9 \%$ 。（P均<0.05）。40 岁组, 女性中心性肥胖发生率高于男性, 但无统计学意义 （男性41.0\% vs. 女性44.1\%，P>0.05）。详见表5。

表5 各年龄组不同性别体检人群中心性肥胖发生情况 [n（\%）]。

\begin{tabular}{llllll}
\hline 年龄组 & 性别 & 非超标者 & 肥胖者 & $\chi^{2}$ & $P$ \\
\hline \multirow{2}{*}{$20^{\sim}$} & 男 & $21(67.7 \%)$ & $10(32.3 \%)$ & \multirow{2}{*}{5.206} & \multirow{2}{*}{0.023} \\
& 女 & $41(91.1 \%)$ & $4(8.1 \%)$ & & \\
30 & 男 & $10(52.6 \%)$ & $9(47.4 \%)$ & \multirow{2}{*}{3.997} & 0.049 \\
& 女 & $37(77.1 \%)$ & $11(22.9 \%)$ & & \\
4 & 男 & $15(59.0 \%)$ & $13(41.0 \%)$ & \multirow{2}{*}{0.033} & 0.856 \\
& 女 & $19(55.9 \%)$ & $15(44.1 \%)$ & & \\
\hline
\end{tabular}

2)不同年龄组体检人群中心性肥胖发生情况

随着年龄的上升, 男性中心性肥胖发生率先升高后降 低, 分别为: $20^{\sim}$ 岁组 $32.3 \%, 30^{\sim}$ 岁组 $47.4 \%, 40^{\sim}$ 岁以上 组 $41.0 \%$, 但差异无显著性 $\left(x^{2}=1.639, \mathrm{P}=0.441\right)$ 。随 着年龄的增长, 女性中心性肥胖发生率逐渐升高, 分别为: $20^{\sim}$ 岁组 $8.9 \%, 30^{\sim}$ 岁组 $22.9 \%, 40^{\sim}$ 岁以上组 $44.1 \%$ $\left(x^{2}=13.343, P=0.001\right)$ 。具体情况如图3所示。

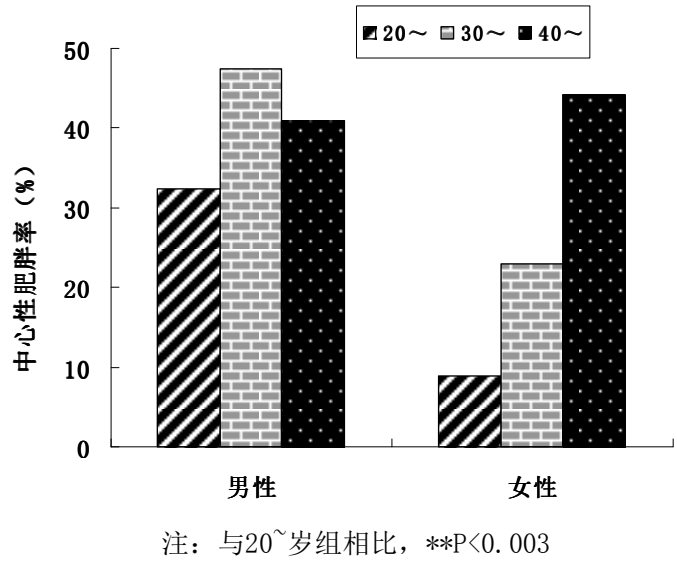

图3 男女体检人群不同年龄组中心性肥胖发生率。

\section{5. 体检人群多项指标的多元线性回归分析}

以体检人群的BMI为因变量, 以部分体成分及血生化、 激素指标为自变量, 建立多元线性回归模型。检验并排除 存在严重多重共线性的自变量, 得到如下线性回归模型:

$\mathrm{Y}=-1.157+0.210 \mathrm{X} 1+0.243 \mathrm{X} 2+0.536 \mathrm{X} 3+0.734 \mathrm{X} 4$, 决定 系数 $R 2=0.872$, 其中:

$\mathrm{Y}-\mathrm{-BMI}\left(\mathrm{Kg} / \mathrm{m}^{2}\right)$

X1--腰围 $(\mathrm{cm})$

X2--体脂肪率（\%)

X3--Lnins

X4--性别（0：女性；1：男性）

各自变量的标准化偏回归系数如表 6 所示:

表6 各自变量的标准化偏回归系数。

\begin{tabular}{lll}
\hline 自变量 & 标化系数 & $\mathrm{P}$ \\
\hline 腰围 $(\mathrm{cm})$ & 0.650 & 0.000 \\
体脂肪率 $(\%)$ & 0.358 & 0.000 \\
Lnins & 0.082 & 0.005 \\
性别 & 0.103 & 0.014 \\
\hline
\end{tabular}

\section{4. 讨论}

\section{1. 不同性别体检人群体成分指标分析}

体成分 [3] (Body Composition) 是构成人体各器官, 组织等的总称。主要由体脂重和去脂体重组成。人体生长 发育状况以及营养状况等因素在一定程度上影响了体成 分。机体内组织与器官的构成按一定的比例, 其与健康关 系密切, 也是评价人体机能水平的重要指标。

林梅 [4] 等的研究显示, 男性肌肉量与身体质量指数 均显著高于女性。郑延松 [5]的研究表明, 男性肌肉量、 去脂体重、去脂质量指数和BMI显著高于女性。而女性体 脂肪率和体脂质量指数显著高于男性。对于BMI正常人群, 女性体脂肪量、体脂肪率、脂肪体重指数也高于男性, 而 去脂体重及去脂体重指数低于男性。王小迪 [6]等人经过 研究, 认为不同性别老年人体成分、血压存在显著差异。 老年男性在身高、体重、去脂体重、肌肉量、水分量、细 胞内液、细胞外液、蛋白质量、腰臀比等指标均显著高于 女性, 而体脂肪率、肥胖率则均显著低于女性, 这一差异 与其他年龄男女体成分差异一致。不同年龄段中, 女性体 脂肪率均显著高于同年龄段男性 [7]。

本研究结果显示, 同年龄组体检人群男性身高, 体重, 腰围, BMI, 水分量百分比, 肌肉量百分比, 矿物质百分 比, 超重率均显著高于女性。而女性在 $40^{\sim}$ 岁年龄组的体 脂肪率显著高于男性。男性在 $20^{\sim}$ 岁以及 $30^{\sim}$ 岁年龄组中心 性肥胖率均显著高于女性, 而至 $40^{\sim}$ 岁年龄组, 女性中心 性肥胖率高于男性, 但差异不显著。这表明, 随着年龄的 增长, 女性更容易产生脂肪堆积, 形成中心性肥胖, 而女 性体脂肪率高于男性这一结论与以上研究相一致, 男女性 体内脂肪、肌肉的含量与激素等因素有关, 女性体内的雌 激素有利于脂肪的堆积, 而男性体内睪酮水平可促进肌肉 的增加。另外, 由于男女性体重有显著性差异, 所以用脂 肪量、腰围等绝对指标来研究男女性肥胖程度以及身体形 
态差异是不够科学, 是体脂肪率、身体质量指数以及各项 体成分指标的相对值更能较好地用于此目的。

\section{2. 不同年龄体检人群体成分指标分析}

不同年龄段进行比较, 男性体脂肪量在 $41^{\sim} 65$ 岁较 $18 \sim 40$ 岁有显著升高。而男女性相比，女性在 $41 \sim 65$ 岁年龄 段腰慰比超标比例为 $80.4 \%$, 而男性仅 $59.1 \%$ 。李宝新 [8] 对各年龄段体成分状况进行研究, 认为随着年龄逐渐增加, 各性别受试者身体各部位脂肪含量逐渐增加, 各部位肌肉 含量逐渐降低。刘瑛等人 $[9]$ 的研究表明, 人体脂肪含量 随着年龄逐年增长，且逐渐呈向心型分布。男性体脂含量 36 岁后开始突增, 且增长速度较快, 而肌肉含量、水分量 则在60岁以后显著减少。对于女性而言, 身体成分（包括 肌肉量, 水分量, 蛋白质含量, 矿物质含量) 等指标随年 龄变化并不明显, 而女性脂肪量、体脂肪率、BMI等指标 虽然随年龄增加而增加, 但并无明显的突增期。董杰 [10] 认为, 1）男性在30-39岁年龄段, 其BMI将到达峰值。而 当年龄到达 40-49岁时, 体脂肪量以及体脂肪率将攀向峰 值。50岁后, 男性体脂肪率与脂肪量将开始衰减。2）女 性BMI 峰值同样出现在 30-39岁年龄段, 而女性40-49岁年 龄段, 体脂肪量显著增加, 体脂肪率也随之增加; 同样, 女性脂肪量和脂肪率在 40-49岁到达峰值。

本研究结果显示, 无论男女, 随着年龄的增大, 平均 身高, 水分量百分比, 肌肉量百分比和蛋白质百分比均逐 渐降低, 矿物质百分比均有降低趋势, 女性的体重, 腰围, 体脂肪率, 体脂肪量, BMI等指标逐渐上升, 男性的平均 体重, 腰围, 体脂肪率, 体脂肪量, BMI等指标有升高趋 势。这与上述研究结果相一致。30岁以后, 体育锻炼减少, 工作压力大、应酬增多、饮食不规律, 且由于该体检人群 大多来自城市, 多为脑力劳动者, 经济状况良好, 体力劳 动较少, 均有可能造成体内脂肪量增加、肌肉量减少, 这 也导致了蛋白质、无机盐等指标下降。这也是该为人群体 脂肪率以及腰围超标率随年龄逐年的原因。

\section{3. 不同性别体检人群血生化和激素指标分析}

瘦素是脂肪细胞分泌的一种脂源性内分泌多肽激素。 其生理作用主要是抑制食欲、减少能量摄取、增加能量消 耗、抑制脂肪合成。影响瘦素分泌的主要因素有: 体内脂 肪含量、年龄、性别、胰岛素等。研究表明, 女性的瘦素 水平几乎是男性的2倍 [11]。目前许多研究结果均指出, 胰岛素和瘦素之间构成了一个双向反馈环, 在不同层面间 相互作用, 并保持动态平衡。血脂含量受生活地区、膳食、 年龄、性别、职业以及代谢等因素的影响, 波动范围较大。 不同年龄, 不同性别, 血脂均有差异。男性高于女性, 女 性LDL-C升高明显低于男性, 而HDL-C升高则是女性多于男 性, 这可能是女性患冠心病少于男性的原因之一 [12]。强 丹 [13] 等人研究表明, 男性组TG、TC、TG、HDL-C高于女 性组, 胰岛素、FBG等指标差异均无统计学意义。有研究 表明 [14], 男性TC、HDL-C和LDL-C水平低于女性。TG水平 高于女性。阴斌霞 $[15]$ 研究表明, 男女性LDL-C有显著性 差异; TG和HDL-C有极显著性差异。
本研究结果显示, 同一年龄组, 男性HDL-C低于女性, LDL-C、TCG、TG、Lnins高于女性，FBG无显著性差异。在 $20^{\sim}$ 岁组和 $40^{\sim}$ 岁组, 男性Lnlep低于女性, $30^{\sim}$ 岁组, 男性 Lnlep高于女性, 提示, 同一年龄段, 女性的血脂和胰岛 素水平均优好于男性, 这可能与男性经常吸烟、饮酒、频 繁应酬有关; 过量饮酒后, 乙醇可对肝微粒体、高尔基体 脂糖蛋白的合成、成熟和释放产生影响, 脂肪在肝中堆积 增加; 加上大量的乙醇本身也提供合成脂肪酸原料, 使进 入肝细胞的脂肪酸量增多, 而导致血脂增高。通常情况下, 女性的瘦素水平高于男性, 这与前人的研究结果相一致, $30 \sim$ 岁组男性瘦素水平高于女性, 分析原因是由于该组男 性人数较少, 可能与真实情况存在差异, 今后应增大样本 量进行研究。

\section{4. 不同年龄体检人群血液激素指标分析}

黄榕等人研究表明 $[11]$, 成年人群中血瘦素随年龄变 化差异不显著。老年组TG、TC、LDL、ApoB明显高于青年 组, 组间比较有显著性差异。俞雅萍 [14]研究表明, 50 岁以上人群 TG和 HDL-C水平高于 50 岁以下人群, 差异显著。 TC、LDL-C水平差异不显著。常永超 [16]等人研究表明, 各年龄段空腹血糖、血脂检测值随着年龄的增长均有所增 高。血清TG在 $40-49$ 年龄段最高, 差别显著。

本研究结果表明, 随着年龄的增加, 男性平均FBG逐 渐升高, TC、 $\mathrm{LDL}-\mathrm{C}$ 有上升趋势, 但差异无显著性; 其平 均 TG、HDL-C、Lnlep、Lnins先下降后上升, 但差异无显 著性。女性随着年龄的增加, 其平均 $F B G$ 逐渐升高, $\mathrm{TG}$ 、 TC、 LDL-C有上升趋势但差异无显著性; 平均Lnlep、Lnins 有下降趋势, 但差异无显著性。提示, 随着年龄的增长, 血液各指标均朝着不利于健康的方向发展, 分析原因可能 与饮食结构和生活习惯的改变有关, 工作之后, 饮酒、应 酬逐渐增多, 长期以往, 血液激素各指标发生改变。

\section{5. 体检人群多项指标多元线性回归分析}

BMI, 又称为体质指数, 计算公式: BMI $=\frac{\text { 体重 }(\mathrm{Kg})}{\text { 身高 }(\mathrm{m})^{2}}$,

它是目前国际上评价人体肥胖程度及营养状况的常用指 标 [17]。1990年, 世界卫生组织 (WHO) 推荐使用BMI作为 肥胖的评价指标。BMI在一系列评价营养或肥胖状况的指 标中最为常用。对于成年人 (年龄 $\geqslant 18$ 周岁者), 使用BMI 评价肥胖状况时将不再区分性别。

一般认为, 成人的BMI越大, 其脂肪含量越高。到达 一定程度将提示超重或者肥胖, 因为超重或肥胖意味着人 体脂肪含量的上升 [18]。季玉珍 [19] 研究显示, 随着BMI 的增加, 无论男女, 大学生的体脂肪 (率) 以及去脂体重 均有所上升。对于腰围、慰围、胸围、上臂紧张围、上臂 松弛围、腰臂比、维尔维克指数、上臂围差等指标均随BMI 增加而逐渐增加。舒画 ${ }^{[7]}$ 等认为, 不同BMI受试者的体水 分量、蛋白质、无机盐及肌肉量等差异显著。体脂肪率随 BMI水平升高而增加。

本研究结果表明, BMI的影响因素有腰围、体脂肪率、 性别和胰岛素的自然对数, 其中腰围对BMI的影响最大, 其次是体脂肪率。BMI主要反映的是人的胖瘦程度和营养 
状况, 而腰围则是中心性肥胖的评价指标, 而体脂肪率则 反映了脂肪成分占体重的百分比, 这些反映人体肥胖或是 营养状况的指标在一定程度上相互影响, 因此在评价人体 体型以及营养程度时，不应仅局限于一种指标，而应该利 用不同的指标进行综合考虑。一般情况下, 由于成年男性 吸烟、饮酒以及应酬较多, 所以更容易肥胖, 因此性别也 有可能对BMI产生影响。另外胰岛素有利于甘油三酯的合 成, 使得脂肪更易于在体内堆积; 或者是在BMI水平高的 人群机体可能出现糖耐量降低或是糖代谢紊乱, 从而导致 胰岛素抵抗, 导致BMI水平高的人群出现高胰岛素血症, 上述情况均可能导致BMI与胰岛素之间成一定程度的正相 关。

\section{5. 结论}

1) 本体检人群同年龄组男性与女性相比，体成分与 血液生化和激素指标均提示男性发生肥胖、血糖血脂紊乱 等代谢性疾病的风险更高; 而随着年龄增长, 体检人群的 各项指标均朝向不利于健康的方向发展, 且女性比男性的 变化更大。

2) 本体检人群BMI的影响因素主要有: 腰围、体脂肪 率、胰岛素的自然对数以及性别。且随着腰围和体脂肪率 的升高, BMI也相应升高, 且男性比女性的BMI升高更明显。 这提示在判断人体肥胖程度时, 可用BMI、体脂肪率以及 腰围等指标进行综合分析, 且对于男性和女性的标准不应 完全一致。

3) 对于本体检人群, BMI与胰岛素的自然对数成一定 程度的正相关, 这可能由于胰岛素促进合成甘油三酯, 使 得机体肥胖程度增加, 从而提高BMI。

\section{参考文献}

[1] 孔灵芝, 陈春明. 中国成人超重和肥胖症预防控制指南 [M]. 北京: 北京人民卫生出版社, 2006:1-35。

[2] 中国成人血脂异常防治指南制订联合委员会. 中国成人血 脂异常防治指南 $[\mathrm{J}]$. 中华心血管病杂 志, 2007, 35 (5) : 390-419。

[3] 门杰, 胡鹏宇, 崔春阳, 等. 体育锻炼对身体成分影响的研 究进展 [J]. 长春师范学院学报, 2014 (02) : 115-118。
［4］林梅, 凌文志, 黄迎春, 等. 门诊不同年龄性别体检者人体 成分调查分析 $[J]$. 医学研究生学报, 2015 (03) : 294-296。

[5] 郑延松, 卢艳慧, 舒画, 等. 中国成人去脂体重指数与脂肪 体重指数分析 $[Z]$. 天津武清: $2011 ， 158-161$ 。

［6］王小迪, 张保国. 不同性别老年人身体成分、血压指标的差 异及相关性 [J]. 中国老年学杂志, 2011 (18) : 3637-3638。

[7] 舒画, 郑延松, 李春霖, 等. 不同体重状态人群人体成分的 对比分析 [J]. 中华医学杂志, 2012, 92 (48) :3412-3416。

［8］李宝新. 不同年龄健康人群不同部位骨密度与体成分分析 [D]. 河北医科大学, 2009。

[9] 刘瑛, 刘艳华, 王小迪. 成年人身体脂肪含量及分布随年龄 变化趋势 $[J]$. 中国老年学杂志, $2013(22): 5713-5715$ 。

[10］董杰. 20 59岁成人身体成分与脂肪分布趋势研究 $[J]$. 内 蒙古民族大学学报 (自然科学 版), 2008, 23 (4): 476-477, 480。

[11] 黄榕, 许力舒. 瘦素及瘦素受体与高血压的研究进展 [J]. 医学综述, 2005 (07) :597-598。

[12] 田伟, 张莉, 陈利, 等. 高原地区健康成人血脂四项正常测 值 $[J]$. 临床军医杂志, 2008(01) : 97-98。

[13] 强丹, 白云贤, 牛旭东, 等. 空腹血糖及血脂与胰岛素抵抗 关系的研究 $[J]$. 宁夏医科大学学报, 2013 (11) : 1237-1239。

[14] 俞雅萍. 绍兴市人群血脂水平性别、年龄及城乡差异 $[J]$. 中国临床康复, 2005 (07) : 72-73。

[15] 阴斌霞, 赵丽华, 耿燕, 等. 西安地区部分健康体检人群血 脂水平分析 $[J]$. 现代检验医学杂志, 2006 (06) : 70-73。

［16］常永超, 江涛, 李维, 等. 不同年龄成年体检者血糖和血脂 的变化特点 $[\mathrm{J}]$. 河南科技大学学报（医学 版), 2009 (02) : 146-147。

[17] Dietz W H, Bellizzi M C. Introduction: the use of body mass index to assess obesity in children[J]. Am J Clin Nutr, 1999, 70 (1) : 123S-125S.

[18] 王步标, 华明. 运动生理学 [M]. 北京: 高等教育出版社, 2006。

[19] 季玉珍. 大学生BMI与体成分和部分素质指标的关系 [D]. 浙 江大学, 2011。 